\title{
Graphene and Carbon Nanotube Auxetic Rubber Bionic Composites with Negative Variation of the Electrical Resistance and Comparison with Their Nonbionic Counterparts
}

\author{
Luca Valentini, * Silvia Bittolo Bon, and Nicola M. Pugno*
}

Prof. L. Valentini Dipartimento di Ingegneria Civile e Ambientale Università di Perugia UdR INSTM Strada di Pentima 4, 05100 Terni, Italy E-mail: luca.valentini@unipg.it

Dr. S. Bittolo Bon Consorzio Interuniversitario per la Scienza e Tecnologia dei Materiali UdR di Perugia Strada di Pentima 4, 05100 Terni, Italy

Prof. N. M. Pugno Laboratory of Bio-Inspired and Graphene Nanomechanics Department of Civil Environmental and Mechanical Engineering University of Trento Via Mesiano 77, I-38123 Trento, Italy E-mail: nicola.pugno@unitn.it

Prof. N. M. Pugno School of Engineering and Materials Science Queen Mary University of London Mile End Road, London E1 4NS, UK

Prof. N. M. Pugno Ket Lab, Italian Space Agency via del Politecnico snc, I-00133 Roma, Italy

\begin{abstract}
Microorganism metabolic activity can facilitate the formation of cellular material systems that have unusual mechanical and physical properties. In the living world microorganisms are commonly used for preparing porous food by fermentation; here carbon nanotubes, graphene nanoplatelets, and a mix of them are dispersed in liquid silicone rubber with single-cell fungi of commercial beer yeast. The fermentation of such microorganisms during the gelling of the silicone matrix results in bionic composites with buckled/collapsed cells that infer, as rationalized with an analytical model and excluded in a abiotic experimental comparison, auxetic properties. During stretching it is found that the Poisson's ratio of such composites changes sign, from negative to positive, and the variation of the electrical resistance is negative. In addition to the conductivity increment, a general increment of the stretchability and damage resistance with respect to the composites prepared by abiotic process is observed. Bionic composites, even if in their infancy, can thus be multifunctional and superior to their traditional/abiotic counterparts.
\end{abstract}

\section{Introduction}

Foams are cellular solids consisting of a mix of open and closed cells where the mechanical properties of the foam are determined by the volume fraction of solid material and the cell structure.[1-4] If we consider isotropic linear elastic materials, then their elastic response is described by the Young's modulus and Poisson's ratio, connected to the shear and bulk modulus. Although the intuitive thought is that elastic materials shrink/expand laterally when stretched/compressed axially (so that the Poisson's ratio is positive), many $2 \mathrm{D}$ and $3 \mathrm{D}$ structures and materials with negative Poisson's ratio have been reported.[1] Several attempts based on traditional/ abiotic processes using 3D polymer templates provided a facile, low-cost, and scalable method for creating nanomaterial-based foams including organic nanomaterials, $[5,6] \mathrm{metal} / \mathrm{metal}$ oxide nanomaterials, $[7,8]$ and carbon-based nanomaterials (e.g., carbon nanotubes (CNTs), graphene).[912] Between various carbon-based nanomaterials, carbon nanotube products, including CNT fibers, CNT films, and CNT aerogels, have been recently developed for carbon-based nanomaterials foams.[13,14] Also the research on polymer composites has yielded interesting results in mechanical properties of nanocomposite foams.[15-17] If we look at the mechanical behavior, most of these materials shrink laterally like a rubber band when stretched, so their Poisson's ratios are positive. Likewise, most materials become thinner in widthwise direction when stretched along their length. Even if most of materials, including foams, exhibit a positive Poisson's ratio, theoretically negative Poisson's ratios (called auxetic behavior) are permissible. In particular, the range of Poisson's ratio for linear elastic isotropic materials is restricted 
between -1 and $+0.5,[18]$ with the upperbound usually describing rubber-like materials, in contrast to the bionic rubber presented in this paper resulting in Poisson's ratio close to the lowerbound. In this regard, in the past there were several studies confirming that CNT films can change in-plane Poisson's ratios from negative to positive during a uniaxial tensile loading,[19] while recently polymer-based graphene foams also exhibit negative Poisson's ratio due to the presence of buckled structures.[20] This auxetic behavior was attributed to the fact that, instead of conventional foams, their cell structure was found to be "re-entrant" (i.e., the cell wall protrude inward rather than outward). Such re-entrant structures were obtained artificially by exploiting the mechanical instabilities through compressive buckling or crumpling of graphene nanoplatelets (GNPs) on stretchable polymer substrates.[21] Nowadays it is a challenge for the researchers to synthesize materials by adding nanomaterials via natural metabolic activities leading to auxetic properties of the synthesized foams. In nature living microorganisms such as yeast cells are commonly used for preparing porous food (e.g., baked bread, cake, etc.) by fermentation. This microorganism inspired method can be used to assembly in fast way hybrid porous nanostructures with natural and ecosustainable reagents. Thus, microorganisms can facilitate the formation of a wide range of porous nanomaterials that have unique physical properties as well as structures that are not produced by abiotic processes. As an example bacteria and fungi were extensively used in materials science as simple templates with monodisperse sizes and controlled shapes as well as scalable growth.[22,23] More recently the metabolic activities of biological processes such as beer fermentation, were adopted to generate porous hierarchical composites in gelling materials during their cross-linking or bionic porous nanocomposites based on intractable polymers. [24-27] Inspired by the idea of bubble migration from the fermentation bath[25] and by the observation that when the $\mathrm{CO} 2$ bubble was released by the yeast fermentation, it deforms once in contact with the liquid medium, we could be able to create re-entrant cell architectures by gelling the deformed bubble in a cross-linking system that changes its viscosity during the vulcanization process. Silicon rubber (SR) is a gelling material obtained by polycondensation or polyaddition reactions; SRs are widely used for a variety of scopes including tissue implants because of their flexibility and chemical stability.[28] Moreover, in several medical implants such as neuronal implants, there is a need to restore their functionalities by electrical stimulation and in this regard carbon nanotube-SR and graphene/SR composites could be considered as promising candidates for use in tissue implants possessing proper mechanical, electrical as well as biocompatible properties.[29-32] Auxetic stents made of such biocompatible composites, for example, could help minimizing the negative effects of current stent designs through tailored negative Poisson's ratio, deformation mechanism and enhanced mechanical properties. The manufacturing of smart filters, that when you pull them their pores become larger, could find application in medication delivery into a swollen wound where the gradual release of the medication is controlled by the pulling force of the wound. Thus, even if the biocompatibility of SR reinforced with nanofillers has still to be demonstrated (this is also outside the aim of the present paper), such new composites, especially when bionic and thus with peculiar properties, could find application even in medicine. Combining features of such natural microorganism process with artificial nanomaterials can lead to design innovative bionic nanocomposites. In the present work, a series of porous composites obtained by microorganism nutrition process were prepared by the simultaneous reactions of beer's yeast fermentation and the gelation of liquid SR mixed with nanostructured carbon materials (i.e., CNT and GNPS). Our analyses demonstrated the presence of buckled/collapsed cell structure in our bionic composites, which results in an extreme auxetic deformation behavior. Also the negative variation of the electrical resistance is interesting and unexpected. These results show that after the formation of buckled/collapsed pore structure, improvement in stretchability and electrical conductivity could be achieved simultaneously in our multifunctional bionic composites.

\section{Results and discussion}


The fermentation assisted method proposed for the realization of our bionic composites involves a prereaction state where the solution containing yeast and sugar releases $\mathrm{CO} 2$ bubbles that are trapped by the cross-linking agent in SR, when the viscosity of the solution starts to increase with the polymerization degree. Once the gelation will start, the accumulation of $\mathrm{CO} 2$ gas, that needs to escape, generates pores and, as a result, porous SR and SR composite have been obtained (Figure $1 a, b)$, respectively. Figure $1 c-f$ shows the images of the bionic SR composites. Considering that the strategy adopted in this work is to reproduce the Saccharomyces cerevisiae through a process called "budding", where a daughter cell is initiated as growth from the mother cell, it is reasonable to suggest that the yeast cells remain trapped in the gelling matrix.[26] As seen in Figure 1c, the yeast cells were completely removed by postthermal annealing at $250{ }^{\circ} \mathrm{C}$ of the bionic composite after their cure. From the field emission scanning electron microscopy (FESEM) analysis it is possible to observe that the cells in the SR/CNTs composite are round and rather symmetrical, similar to conventional cell structure previously reported for SR foams (Figure 1d).[1] In contrast, the cells of the bionic SR/GNPs and SR/CNTs/ GNPs composites, shown in Figure 1e,f, are collapsed or buckled. The values of the pore size and the pore shape (i.e., circularity that is a measure of how circular each particle is; i.e., particles are modeled with ellipses: an ellipse with a circularity of 0 is a straight line, while an ellipse with a circularity of 1 is a perfect circle) as well as the statistics from the analysis of the cross sections of the bionic samples shown in Figure $1 c-f$ are reported in Table S3 and Figure S2 (Supporting Information), respectively. From these data it is evident that the addition of GNPs in the bionic composites makes the pores less circular with a collapsed or buckled morphology. This finding could be explained according to a model recently proposed by Valentini et al.[25], where the assembly of GNPs at liquid- liquid immiscible interface (water and silicone in this case) is driven by interfacial tension of the bubble shell that is producing during the fermentation and that can be estimated as $\gamma w \pm \sigma G N P s t G N P s$ where $\gamma w$ is the water surface tension $\left(71 \mathrm{mN} \mathrm{m}-1\right.$ at $25^{\circ} \mathrm{C}$ ), $\sigma G N P s$ is the film stress and tGNPs is the thickness of the retracting film once the bubble comes in contact with water/silicone interface. Thus the bubble radius deformation can be considered directly proportional to stress in the coating times its thickness ("interfacial tension"). The interfacial tension is thus the main responsible for the bubble deformation and for the final collapsed structure once the gelling is occurring. Moreover, accordingly to the results presented in ref. [25] the stress of the GNP film was found to be higher than that observed for CNTs, and this takes into account the observed collapsed structures in the bionic SR/GNPs and SR/ CNTs/GNPs composites. Moreover, the response of the bionic SR/CNTs/GNPs sample (Figure 2a inset) to uniaxial tensile strain exhibits lateral expansion, which is the typical behavior of negative Poisson's ratio effect. Figure 2a shows the relationship between the in-plane (i.e., measured by the contraction of the width) Poisson's ratio and the strain of the prepared bionic samples. When the tensile strain was small $\approx 7 \%$ for bionic SR, bionic SR/GNPs, or bionic SR/CNTs/GNPs samples, respectively, the Poisson's ratio was negative. With the increased strain, the Poisson's ratio also increased and became positive gradually. Similar to that of conventional SR material, the Poisson's ratios of bionic SR/CNTs composite are positive and near +0.15 at small applied tensile strain level, maintaining this value also at high strain level. The negative Poisson's ratio of our composites sharply contrasts with that reported in Figure $2 b$ for conventional SR composites where the experimentally observed Poisson's ratios are positive. An analytical model was developed that predicts the observed sign change of the Poisson's ratios for bionic composites under large strain. This model takes into account key structural features of our bionic composites: (i) isotropic matrix properties; (ii) buckled/collapsed porosity with variable size at small strains and (iii) even shape at large strains. For a material stretched with a length increase in the stretching direction the relative change of volume $(\Delta V / V)$ is (see Supporting Information)

$$
\begin{aligned}
\Delta V / V= & \exp \left((1-2 v)^{*} \varepsilon\right)-1 \\
= & p^{*}\left(\exp \left(\left(1-2 v_{p}\right)^{*} \varepsilon\right)-1\right) \\
& +(1-p)^{*}\left(\exp \left(\left(1-2 v_{m}\right) * \varepsilon\right)-1\right), \\
p= & p_{0} * \exp (-\beta * \varepsilon)
\end{aligned}
$$


where $p$ is the pore volume fraction, vm is the Poisson's ratio of the matrix, $p 0$ is the pore volume fraction at vanishing strain $(\varepsilon=0)$, and $\beta$ is a shape factor governing the variation of the pore shape with strain. Equation (1) for $\varepsilon \rightarrow 0$ and for "Hoberman sphere"-like pores (vp $=-1$, Figure $2 a$ inset and proof in the Supporting Information) gives

$$
v=\left(1-3 p-(1-p) *\left(1-2 * v_{\mathrm{m}}\right)\right) / 2
$$

that restitutes for $p=0$ the Poisson's ratio of the matrix $(v m)$ while for $p=1$ the Poisson ratio of the pores, here equal to -1 . For $\varepsilon \rightarrow \infty$, Equation (2) gives the Poisson's ratio of the matrix (i.e., vm). The same selfconsistent asymptotic behaviors are guaranteed by Equation (1) for $\beta>3$. Using for fitting the experimental results the model of Equation (2) (similar behaviors are obtained using the more complex "Poisson's mixture rule" of Equation (1)), the dependence of the Poisson's ratios with the strain has been predicted for our bionic composites for specific $\beta, \mathrm{p} 0$, and vm parameters as reported in Figure $2 \mathrm{c}$. In particular, the model predicts the observed Poisson's ratios for the bionic SR and bionic SR/ CNTs/GNPs composite with the best fitting values of $\beta, p 0$, and vm parameters reported in Figure $2 c$. The predicted pore volume fraction and the matrix Poisson's ratio values are comparable with those of vm for $\varepsilon \rightarrow \infty$ and $p 0$ directly measured as reported in, Table S1 (Supporting Information) where p0 was expressed as $1-(\mathrm{Vm} / \mathrm{V})$ being $\mathrm{Vm} / \mathrm{V}$ the ratio between the matrix volume and the total volume of the bionic composites. This analytical model is consistent with the experimental data reported in Table S4 (Supporting Information) where for pores with the lower circularity (and thus with buckled or collapsed structure) a negative value of the Poisson's ratio at $0 \%$ of strain was associated. More in general the Poisson's ratios are lower as the circularity is lower. Figure 3a shows the mechanical characteristics obtained by tensile test of conventional or bionic SR composites, respectively. From the comparison with conventional SR composites, it is evident that the tensile strength is generally reduced by the presence of the pores in the bionic composites (Table 1 ) while the specific strength (i.e., the tensile strength normalized by the density) was generally found to be independent by the pore volume fraction and thus also pore stress concentration. Moreover the stretchability expressed in terms of deformation at break values obtained for the bionic samples, is higher than the respective data obtained for conventional SR composites. The mechanical properties reported above can be rationalized in terms of the agglomeration of the nanophase(s); after defining $\mathrm{V}$ as the total volume of the composite and $\mathrm{v}$ as the (total) volume of the phase (s), it is possible to define $f$ as the (total) volume percentage of the phase(s) referring to the total volume $V$, i.e., $f=v / V$ as reported in Table. 1 . If an ideal dispersion, thus without agglomeration, is considered, the composite mechanical resistance would depend linearly on the percentage of the phase itself, thus an increment of $f$ would cause an increment also in the mechanical resistance (assuming the strength of the inclusion much larger than that of the matrix). This is not true if the agglomeration of the phase takes place, which causes at a given point a decrease of the total resistance as observed in Figure 3e.[33] The mechanical efficiency of the reinforcement was estimated as the ratio of the mean strength of the phase(s) on and of the matrix om by using the direct rule of mixture, namely,

$$
\sigma_{\mathrm{n}} / \sigma_{\mathrm{m}}=\sigma\left(f \sigma_{\mathrm{m}}\right)-(1-f) / f
$$

where $\sigma$ is the mechanical resistance of the composite. The data were reported in Table 1 including the volume fractions. From Table 1 and the mechanical efficiency values, we deduce a synergistic effect for conventional composites of CNTs and GNPs that is maximum for their weight fraction ratio of $0.5 / 0.5$ wt\%, whereas a nearly doubled maximal efficiency for 0.5 wt\% of GNPs (in absence of CNT) for the bionic ones. The increase of elongation at break can be attributed to a decrease in film density (Table S1, Supporting Information) and thus to an increase of the mean free volume of the film.[34] As reported in Table S2 (Supporting Information), the lower bulk density values of the bionic composites is indicative of the free volume between the polymer chains leading to greater chain mobility; a bionic composite would therefore be less resistant and would deform at a lower force than the respective conventional composite. Hence, lower tensile strength and higher elongation is expected for the bionic sample as reported in Table 1 . The 
tensile test results could be rationalized assuming a pullout model[35-37] representing the failure mechanisms that could be related to the high moduli of CNT (GNP) particles, and to the fact that the CNTs (GNPs) make the pores stiffer by interconnection as reported in Figure $3 f$ and Figure S3 (Supporting Information). To explore the effects of the auxetic pore in our bionic composites on their functional performance, we further investigate the electrical characterizations of our samples in response to mechanical deformation. The resistance variations of our samples were studies as a function of tensile strain as shown in Figure 3g. The general electrical resistance increase with extension observed in the conventional SR composites may be explained, according to the model proposed previously by Yamaguchi et al.,[38] by considering the conducting fillers (e.g. CNTs and GNPs) as aggregates regularly spaced that under a homogenous strain would deform in a regular manner, as a result, the electrical resistivity would increase with extension in the direction of strain as the aggregates moved apart. The resistance increase $\Delta R / R O$ of conventional silicone rubber composites is much higher (i.e., $4500 \%$ at $60 \%$ strain for the SR/CNTs/GNPs composite) than that of the analogue bionic composite (i.e., $7.4 \%$ at $90 \%$ strain). It means that the auxetic pores in the bionic composite preserve the sample from the interruption of the conducting network and from a significant decay in conductivity under stretching. Thus, combining with the mechanical performance (e.g., the improved stretchability), the auxetic bionic composites would effectively improve their electrical performance with respect their abiotic (nonbionic) counterparts especially under large strains and thus extreme conditions. Interestingly for the bionic SR/GNPs and SR/CNTs/GNPs composites we observe negative $\Delta R / R 0$ values at larger strain. One possible reason for the negative variation in the electrical resistance at larger extensions may be a net reduction in the distance between complex structures of conducting aggregates which are not spherical in shape and that align under large strains. As reported by Yamaguchi et al.,[38] these rotations may result in a significant alignment of the conducting aggregates, as sketched in Figure $3 \mathrm{~g}$, and are responsible for the negative variation in the electrical resistance with extension in the direction of strain. Finally, it was found that the bionic SR composites with sign change of the Poisson's ratio showed a higher crack resistance than that of the SR composites with positive Poisson's ratio. As reported in Figure 4a V-notch on auxetic bionic SR composite creates a crack blunting thus delaying the crack propagation in the sample and increasing the fracture toughness (see Table $2 \mathrm{~S}$ ). Consequently, the auxetic behaviors preserve to our bionic composites more than $100 \%$ stretchability even after a $\mathrm{V}$ notching, which is important for practical applications of stretchable conductors.

\section{Conclusion}

In this work, we report a biogenic successful method in transforming conventional silicone rubber composites to auxetic robust rubbers. This method is based on carbon dioxide gas generated during yeast fermentation and the gelation process of the liquid rubber matrix. Composites with buckled/collapsed cell structure exhibited an auxetic behavior and a sign change of the Poisson's ratios from negative to positive values under large strains being more stretchable than the corresponding conventional composites. We propose a model for the auxetic properties of our bionic composites, able to capture the observations. Additionally, these bionic composites offer negative electrical resistance change under strain and superior toughness even if less dense. Through such unexpected multifunctional material properties, we could envisage their utilization in macroscopic structures where the negative Poisson's ratio, the negative variation of the electrical resistance, superior stretchablity, and damage resistance with reduced density are ideal multifunctional properties for extreme applications in lighter composites.

\section{Experimental section}

GNPs were kindly supplied by NANESA (G3Nan average thickness of $9 \mathrm{~nm} \approx 25$ layers, bulk density $0.018-$ $0.023 \mathrm{~g} \mathrm{~cm}-3$, average lateral particle size $15 \mu \mathrm{m}$ ). CNTs (NC 7000) were purchased from Nanocyl and their 
structure was confirmed by transmission electron microscopy (bulk density $0.066 \mathrm{~g} \mathrm{~cm}-3$, average diameter $9.5 \mathrm{~nm}$, average length $1.5 \mu \mathrm{m}$ ). S. cerevisiae-based commercial beer yeast extract was used as the medium for fermentation. Crystal liquid rubber (CRISTAL RUBBER purchased from PROCHIMA, density $1.04 \mathrm{~g} \mathrm{~cm}-3$ ) was used for casting with a cold cure by polyaddition. Before using, the rubber $10 \mathrm{wt} \%$ of PT-CURE catalyst (purchased from PROCHIMA, density $1.04 \mathrm{~g} \mathrm{~cm}-3$ ) was added. GNPs or/and CNTs were dispersed in liquid silicone rubber ( $1 \% \mathrm{wt})$ through the utilization of a magnetic stirrer (500 rpm for $3 \mathrm{~h}$ ) to facilitate their dispersion. After that, yeast ( 0.1 weight ratio with respect to the liquid silicone rubber) and sugar (i. e., sucrose, 0.04 weight ratio with respect to the liquid silicone rubber) were previously dispersed in $2 \mathrm{~mL}$ of water, heated at $50{ }^{\circ} \mathrm{C}$ to start the fermentation and added to the silicone mixture. The silicone mixture added with yeast was heated at $50{ }^{\circ} \mathrm{C}$ and the catalyst was added. Then once the fermentation process stopped the polyaddition reaction was completed in an aluminum mold for $24 \mathrm{~h}$ at room temperature. The same procedure was repeated without the addition of the yeast and sugar to prepare SR and SR composites for a comparison purpose. FESEM was used to investigate the cross section of the samples obtained by fracture in liquid nitrogen. The pores size and their shape in terms of circularity were measured through the image analysis software Image-J. The tensile properties of the prepared samples were measured using a universal tensile testing machine (Lloyd Instr. LR30K) with a $500 \mathrm{~N}$ static load cell. The film samples were cut into strips $(100 \mathrm{~mm} \times 10 \mathrm{~mm} \times 3 \mathrm{~mm})$. The gauge length was $60 \mathrm{~mm}$, and the extension rate was set at 10 $\mathrm{mm}$ min-1. Five samples for each composition were tested. The in plane Poisson's ratios of the samples were initially determined by using a micrometer (Borletti, accuracy $0.01 \mathrm{~mm}$ at $20^{\circ} \mathrm{C}$ ) to measure the change in sample width as a function of stretch in the sample length direction. More accurate Poisson's ratio measurements resulted from strain gauge positioned transversal to the strain direction. The fracture toughness was calculated by V-notching the samples in the middle. According to ASTM E23, the standard specimen used had a $2 \mathrm{~mm}$ deep notch with a tip radius of $0.25 \mathrm{~mm}$ machined on one face. Five samples for each composition were tested with the stress-strain curves reported in the Supporting Information. For an infinite sheet with a lateral crack of length, a, subjected to a uniform stress $\sigma$ the stress intensity factor is given by $\mathrm{KI}=\sigma^{*} \mathrm{f}(\mathrm{a} / \mathrm{W})^{*}\left(\pi^{*} \mathrm{a}\right) 1 / 2$ where $\mathrm{f}(\mathrm{a} / \mathrm{W})$ is the geometry factor, $\mathrm{W}$ is the sample width and thus the fracture toughness $\mathrm{KIC}$ is calculated as $\mathrm{KI}(\sigma=\sigma \mathrm{C})=\mathrm{KIC}$ with $\sigma \mathrm{C}$ fracture strength. Defining $\mathrm{a} / \mathrm{W}=\alpha$, the geometry factor for a lateral crack is given by $f(a / W)=0.265^{*}(1-\alpha)^{\wedge} 4+\left(0.857+0.265^{*} \alpha\right) /(1-\alpha)^{\wedge} 1.5$. For the electromechanical tests, the ends of the samples were connected by copper wires. During the tensile process, the electrical resistance (Keithley 4200 SCS under a bias of $1 \mathrm{~V}$ ) was recorded simultaneously.

\section{Acknowledgements}

N.M.P. was supported by the European Research Council (ERC PoC 2015 SILKENE No. 693670) and by the European Commission H2020 under the Graphene Flagship (WP14 "Polymer composites," No. 696656) and under the FET Proactive ("Neurofibres" No. 732344). L.V. and N.M.P. had the idea, supervised the entire research and analyzed the results. L.V. designed and supervised the experimental activity whereas N.M.P. developed the theoretical model. S.B.B. prepared the samples and performed the morphological, mechanical, and electrical characterizations. All authors wrote the papers and have revised and given their approval to the final version of the manuscript. SERMS srl (Terni - Italy) is acknowledged for supporting with FESEM characterization and analysis.

\section{Conflict of Interest}

The authors declare no conflict of interest. 


\section{Keywords}

bionic composites, electrical properties, graphene, mechanical properties

\section{References}

[1] K. E. Evans, A. Alderson, Adv. Mater. 2000, 12, 617.

[2] M. F. Ashby, Met. Trans. 1983, 14A, 1755.

[3] L. J. Gibson, M. F. Ashby, G. S. Schajer, C. I. Robertson, Proc. R. Soc. A 1982, 382, 25.

[4] G. N. Greaves, A. Greer, R. S. Lakes, T. Rouxel, Nat. Mater. 2011, 10, 823.

[5] X. Chen, Adv. Mater. 2012, 24, 5884.

[6] S. Seok, ACS Appl. Mater. Interfaces 2015, 7, 4699.

[7] C. Wu, L. Fang, X. Huang, P. Jiang, ACS Appl. Mater. Interfaces 2014, 6, 21026.

[8] H. F. Pan, ACS Appl. Mater. Interfaces 2015, 7, 101.

[9] H. B. Yao, Adv. Mater. 2013, 25, 6692.

[10] Q. Liang, Adv. Mater. 2015, 27, 4634.

[11] L. Lu, Angew. Chem. 2015, 127, 15380.

[12] Z. Niu, W. Zhou, X. Chen, J. Chen, S. Xie, Adv. Mater. 2015, 27, 6002.

[13] T. Q. Tran, Z. Fan, P. Liu, H. M. Duong, Asia Pac. Confed. Chem. Eng. Congr. 2015, 2015, 2444.

[14] A. Mikhalchan, Z. Fan, T. Q. Tran, P. Liu, V. B. C. Tan, T.-E. Tay, H. M. Duong, Carbon 2016, 102, 409.

[15] K. Y. Chun, Y. Oh, J. Rho, J. H. Ahn, Y. Y. Kim, H. R. Choi, S. Baik, Nat. Nanotechnol. 2010, 5, 853.

[16] J. Suhr, N. Koratkar, P. Keblinski, P. Ajayan, Nat. Mater. 2005, 4, 134.

[17] D. J. Lipomi, M. Vosgueritchian, B. C. K. Tee, S. L. Hellstrom, J. A. Lee, C. H. Fox, Z. N. Bao, Nat. Nanotechnol. 2011, 6, 788.

[18] Y. C. Fung, Foundations of Solid Mechanics, Prentice-Hall, Englewood Cliffs, NJ 1968, p. 353. [19] L. J. Hall, V. R. Coluci, D. S. Galvão, M. E. Kozlov, M. Zhang, S. O. Dantas, R. H. Baughman, Science 2008, 320, 504.

[20] Z. Dai, Sci. Rep. 2016, 6, 32989.

[21] J. Zang, Nat. Mater. 2013, 12, 321.

[22] F. Xia, L. Jiang, Adv. Mater. 2008, 20, 2842.

[23] B. Wang, P. Liu, W. G. Jiang, H. H. Pan, X. R. Xu, R. K. Tang, Angew. Chem., Int. Ed. 2008, 47, 3560.

[24] Q. Zhang, B. Chen, L. Tao, M. Yan, L. Chen, Y. Wei, RSC Adv. 2014, 4, 32475.

[25] L. Valentini, S. Bittolo Bon, N. M. Pugno, ACS Appl. Mater. Interfaces 2016, 8, 22714.

[26] L. Valentini, S. Bittolo Bon, M. Tripathi, E. Iacob, S. Signetti, N. M. Pugno, Sci. Rep. 2016, 6, 27031.

[27] L. Valentini, S. Bittolo Bon, N. M. Pugno, ACS Appl. Mater. Interfaces 2016, 8, 7607. 
[28] M. Matsuoka, T. Akasaka, T. Hashimoto, Y. Totsuka, F. Watari, Biomed. Mater. Eng. 2009, 19, 155.

[29] K. Tegtmeier, P. Aliuos, J. Stieghorst, M. Schickedanz, F. Golly, H. Zernetsch, B. Glasmacher, T. Doll, Phys. Status Solidi A 2014, 211, 1439.

[30] H. Ai, H. Meng, I. Ichinose, S. A. Jones, D. K. Mills, Y. M. Lvov, X. Qiao, J. Neurosci. Methods 2003, 128, 1.

[31] H. S. Dong, S. J. Qi, Biosurf. Biotribol. 2015, 1, 229.

[32] K. Tegtmeier, P. Aliuos, T. Lenarz, T. Doll, Phys. Med. 2016, 1, 8.

[33] N. M. Pugno, R. S. Ruoff, Philos. Mag. 2004, 27, 2829.

[34] L. Yang, A. T. Paulson, Food Res. Int. 2000, 33, 571.

[35] N. M. Pugno, J. Mech. Phys. Solids 2010, 58, 1397.

[36] N. M. Pugno, Nanotechnology 2006, 17, 5480.

[37] X. Shi, B. Peng, N. M. Pugno, Appl. Phys. Lett. 2012, 100, 191913.

[38] K. Yamaguchi, J. J. C. Busfield, A. G. Thomas, J. Polym. Sci., Part B: Polym. Phys. 2003, 41, 2079. 

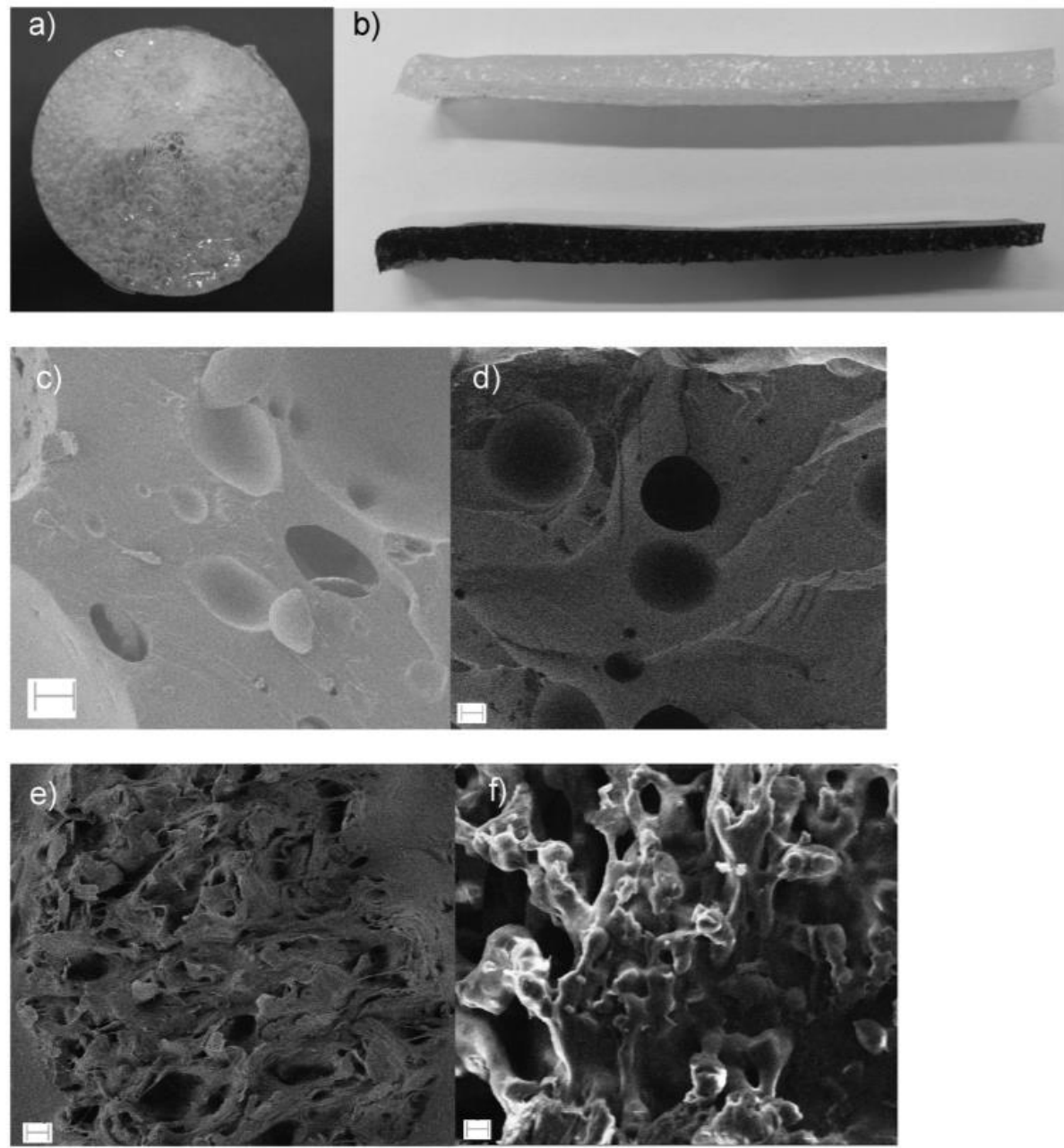

Figure 1. Photoimages of a) circular shaped bionic SR and b) bionic SR (top) and bionic SR/GNP composite (1 wt\%; bottom) prepared by fermentation of yeast in gelling silicone rubber. c-f) Cross section SEM images of representative cell structures for c) bionic SR, d) bionic SR/CNTs (1 wt\%), e) bionic SR/GNPs (1 wt\%), and f) bionic SR/CNTs/GNPs (0.5/0.5 wt\%) composites, respectively. The scale bars indicate $50 \mu \mathrm{m}$. 

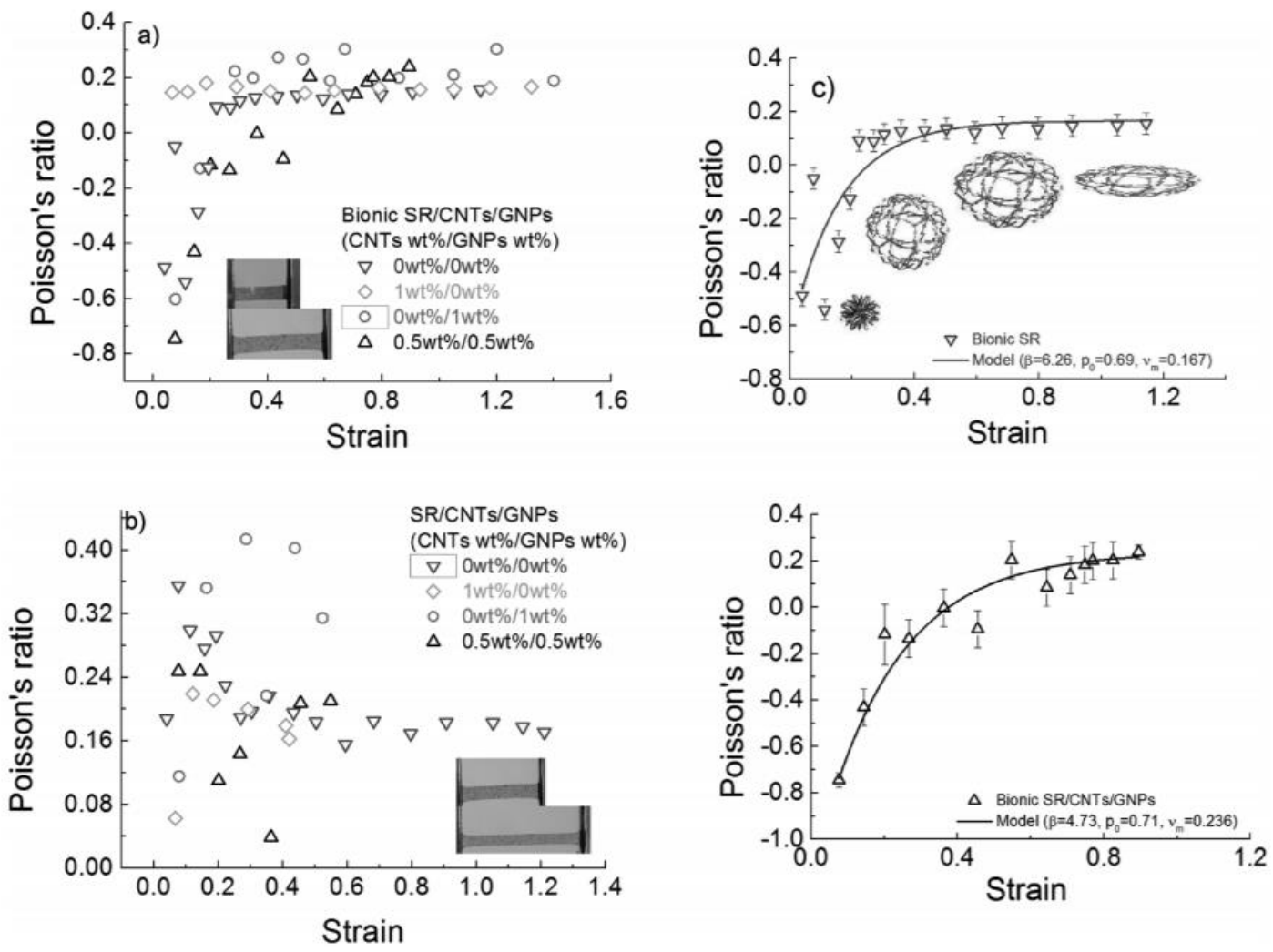

Figure 2. a) Measured Poisson's ratio versus strain for bionic composites. Inset: digital picture of bionic SR/CNTs/GNPs composite under 0 and $\approx 30 \%$ strain. b) Measured Poisson's ratio versus strain for conventional composites. Inset: digital picture of conventional SR/CNTs/CNPs composite under 0 and $\approx 30 \%$ strain. c) Comparison between measured and predicted Poisson's ratio values for bionic SR and bionic SR/CNTs/GNPs (0.5/0.5 wt $\%$ ) composite, respectively. The inset shows the educational toy that is the expanding Hoberman sphere, taken as inspiration for modeling the auxetic porosity.
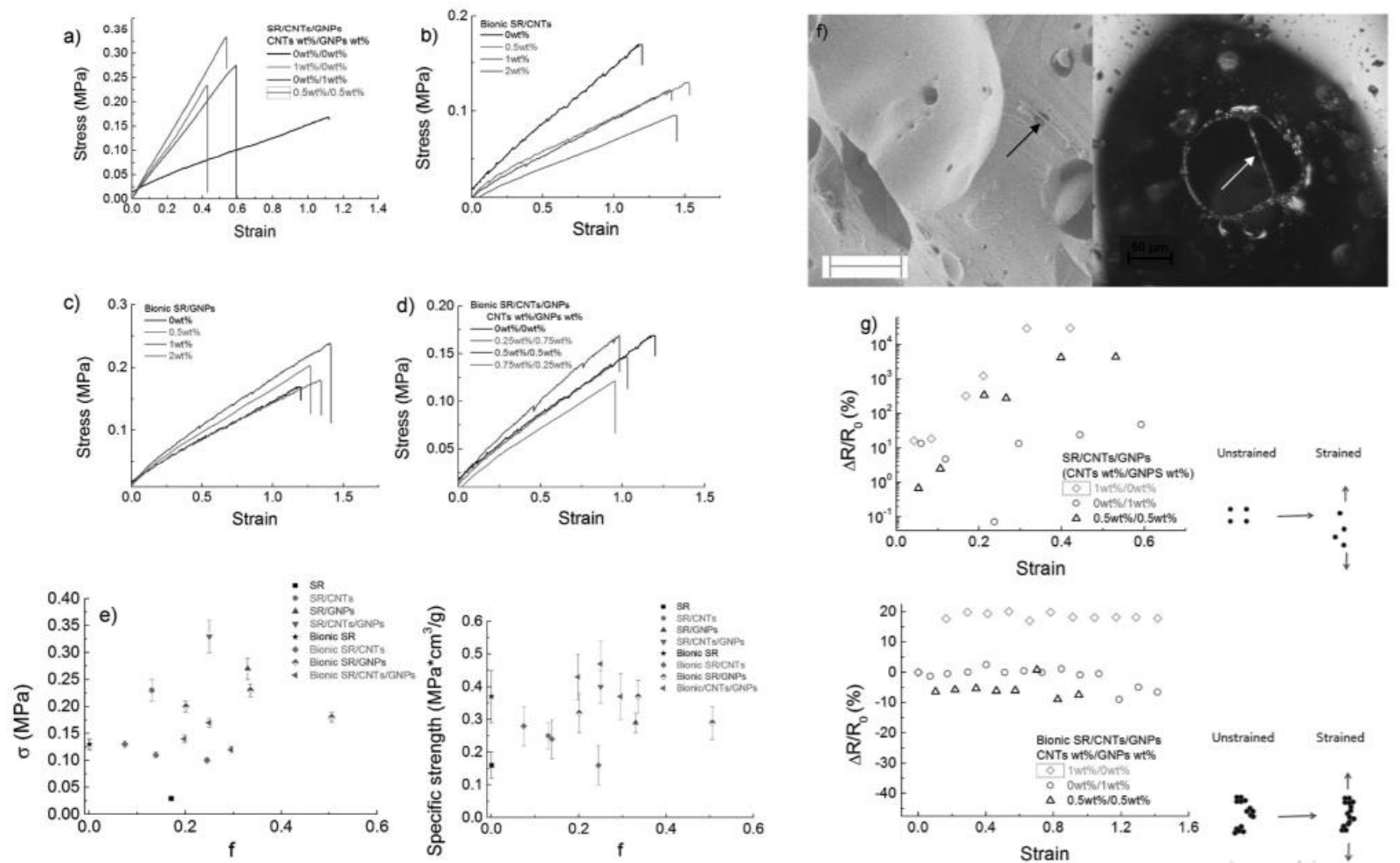

Figure 3. Measured tensile stress of a) conventional SR composites and b-d) bionic SR composites. e) Experimental values of strength $\sigma$ (specific strength) versus volume fraction for a single phase and double phase nonbionic and bionic SR composites. f) SEM and optical images showing cross-sectional view of bionic SR/CNTs (1/0 wt\%) composite where CNTs can be seen bridging between the SR and the cells. The arrows indicate the CNTs. g) Variation of normalized resistance as a function of tensile strain for the prepared samples. Schematic of the movement and orientation under load of aggregates evenly spaced in conventional SR composites (top panel) or anisotropic randomly spaced in bionic composites (bottom panel). 
Table 1. Mechanical characteristics of the prepared samples.

\begin{tabular}{|c|c|c|c|c|c|c|c|}
\hline $\begin{array}{l}\text { Samples } \\
\text { [CNTs wt\%/GNPs wt\%] }\end{array}$ & $\begin{array}{l}\text { Volume } \\
\text { fraction } f\end{array}$ & $\begin{array}{l}\text { Young modulus } \\
\text { [MPa] }\end{array}$ & $\begin{array}{l}\text { Tensile strength } \\
\text { [MPa] }\end{array}$ & $\begin{array}{l}\text { Toughness } \\
\text { [MPa] }\end{array}$ & $\begin{array}{l}\text { Specific strength } \\
{\left[\mathrm{MPa}^{*} \mathrm{~cm}^{3} \mathrm{~g}^{-1}\right]}\end{array}$ & $\sigma_{\mathrm{n}} / \sigma_{\mathrm{m}}$ & $\begin{array}{l}\text { Deformation at break } \\
{[\%]}\end{array}$ \\
\hline $0 / 0$ & 0 & $0.13 \pm 0.01$ & $0.17 \pm 0.03$ & $0.09 \pm 0.01$ & $0.16 \pm 0.04$ & - & $112 \pm 4$ \\
\hline $1 / 0$ & 0.13 & $0.56 \pm 0.03$ & $0.23 \pm 0.02$ & $0.05 \pm 0.01$ & $0.25 \pm 0.04$ & 5.31 & $43 \pm 4$ \\
\hline $0 / 1$ & 0.33 & $0.46 \pm 0.02$ & $0.27 \pm 0.02$ & $0.08 \pm 0.01$ & $0.29 \pm 0.03$ & 2.68 & $59 \pm 3$ \\
\hline $0.5 / 0.5$ & $0.07 / 0.20$ & $0.60 \pm 0.03$ & $0.33 \pm 0.03$ & $0.09 \pm 0.01$ & $0.40 \pm 0.05$ & 6.50 & $54 \pm 2$ \\
\hline \multicolumn{8}{|l|}{ Bionic } \\
\hline $0 / 0$ & 0 & $0.13 \pm 0.01$ & $0.18 \pm 0.02$ & $0.10 \pm 0.01$ & $0.37 \pm 0.08$ & - & $118 \pm 4$ \\
\hline $0.5 / 0$ & 0.07 & $0.05 \pm 0.01$ & $0.13 \pm 0.02$ & $0.10 \pm 0.01$ & $0.28 \pm 0.06$ & 5.05 & $152 \pm 14$ \\
\hline $1 / 0$ & 0.13 & $0.06 \pm 0.01$ & $0.11 \pm 0.02$ & $0.07 \pm 0.01$ & $0.24 \pm 0.06$ & 2.08 & $120 \pm 15$ \\
\hline $2 / 0$ & 0.24 & $0.06 \pm 0.01$ & $0.10 \pm 0.02$ & $0.07 \pm 0.01$ & $0.16 \pm 0.06$ & 0.43 & $144 \pm 10$ \\
\hline $0 / 0.5$ & 0.20 & $0.12 \pm 0.01$ & $0.20 \pm 0.02$ & $0.13 \pm 0.01$ & $0.32 \pm 0.06$ & 2.59 & $126 \pm 12$ \\
\hline $0 / 1$ & 0.33 & $0.16 \pm 0.01$ & $0.23 \pm 0.02$ & $0.16 \pm 0.02$ & $0.37 \pm 0.05$ & 2.16 & $144 \pm 11$ \\
\hline $0 / 2$ & 0.50 & $0.13 \pm 0.01$ & $0.18 \pm 0.02$ & $0.12 \pm 0.01$ & $0.29 \pm 0.05$ & 1.13 & $133 \pm 15$ \\
\hline $0.25 / 0.75$ & $0.03 / 0.27$ & $0.12 \pm 0.01$ & $0.12 \pm 0.02$ & $0.06 \pm 0.01$ & $0.37 \pm 0.07$ & 2.32 & $95 \pm 14$ \\
\hline $0.5 / 0.5$ & $0.07 / 0.20$ & $0.16 \pm 0.01$ & $0.17 \pm 0.02$ & $0.07 \pm 0.01$ & $0.47 \pm 0.10$ & 3.51 & $96 \pm 8$ \\
\hline $0.75 / 0.25$ & $0.10 / 0.11$ & $0.14 \pm 0.01$ & $0.14 \pm 0.02$ & $0.07 \pm 0.01$ & $0.43 \pm 0.08$ & 3.89 & $102 \pm 12$ \\
\hline
\end{tabular}
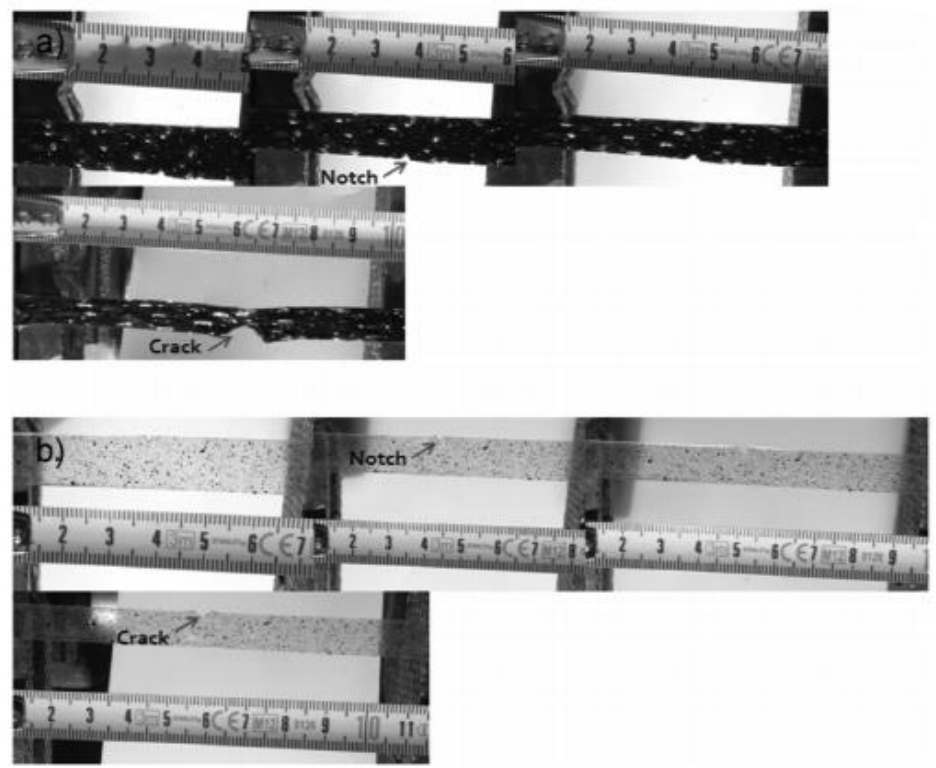

Figure 4. Optical images showing the crack propagating mechanism of a) bionic SR/CNTs/GNPs $(0.5 / 0.5$ wt $\%)$ and b) conventional SR/CNTs/ GNPs $(0.5 / 0.5$ wt $\%)$ composites. 


\section{FUNCTIONAL MATERIALS}

\section{Supporting Information}

for Adv. Funct. Mater., DOI: 10.1002/adfm.201606526

Graphene and Carbon Nanotube Auxetic Rubber Bionic Composites with Negative Variation of the Electrical Resistance and Comparison with Their Nonbionic Counterparts

Luca Valentini, * Silvia Bittolo Bon, and Nicola M. Pugno* 


\section{WILEY-VCH}

\section{Supporting Information}

Graphene and carbon nanotubes auxetic rubber bionic composites, with negative variation of the electrical resistance and comparison with their non-bionic counterparts

Luca Valentini,'* Silvia Bittolo Bon, Nicola M. Pugno*

\section{Analytical Model}

For a material stretched with a length increase of $\Delta \mathrm{L}=\mathrm{L}^{\prime}-\mathrm{L}$ in the stretching direction the relative change of volume $(\Delta \mathrm{V} / \mathrm{V})$ is:

$\Delta \mathrm{V} / \mathrm{V}=\left(\mathrm{L}^{\prime} / \mathrm{L}\right)^{\wedge}(1-2 v)-1$

Eq.1S

from where, introducing the strain as $\varepsilon=\ln \left(\mathrm{L}^{\prime} / \mathrm{L}\right)$, we obtain:

$\Delta \mathrm{V} / \mathrm{V}=\exp \left((1-2 v)^{*} \varepsilon\right)-1$

Eq.2S

Defining $\mathrm{V}_{\mathrm{m}}$ and $\mathrm{V}_{\mathrm{p}}$ as the volumes of the matrix composite and pores, respectively, and with $\mathrm{p}$ the pore volume fraction, we have:

$\Delta V / V=\left(\Delta \mathrm{V}_{\mathrm{m}}+\Delta \mathrm{V}_{\mathrm{p}}\right) / \mathrm{V}=\Delta \mathrm{V}_{\mathrm{m}} / \mathrm{V}+\Delta \mathrm{V}_{\mathrm{p}} / \mathrm{V}=\Delta \mathrm{V}_{\mathrm{m}} /\left(\mathrm{V}_{\mathrm{m}} /(1-\mathrm{p})\right)+\Delta \mathrm{V}_{\mathrm{p}} /\left(\mathrm{V}_{\mathrm{p}} / \mathrm{p}\right)$

Eq.3S

that gives:

$\Delta \mathrm{V} / \mathrm{V}=\mathrm{p} *\left(\Delta \mathrm{V}_{\mathrm{p}} / \mathrm{V}_{\mathrm{p}}\right)+(1-\mathrm{p})^{*} \Delta \mathrm{V}_{\mathrm{m}} / \mathrm{V}_{\mathrm{m}}$ Eq.4S

Thus, according to Eq. $2 \mathrm{~S}$, the relative volume change of the matrix and its pores can be expressed, respectively, as

$\Delta \mathrm{V}_{\mathrm{m}} / \mathrm{V}_{\mathrm{m}}=\exp \left(\left(1-2 v_{\mathrm{m}}\right) * \varepsilon\right)-1$ Eq.5S

$\Delta \mathrm{V}_{\mathrm{p}} / \mathrm{V}_{\mathrm{p}}=\exp \left(\left(1-2 v_{\mathrm{p}}\right)^{*} \varepsilon\right)-1$ Eq.6S 


\section{WILEY-VCH}

where $v_{\mathrm{m}}$ and $v_{\mathrm{p}}$ are the Poisson's ratios of the matrix and pores, respectively.

Including Eqs. 5S, 6S and 2S in Eq. 4S we obtain:

$\Delta \mathrm{V} / \mathrm{V}=\exp \left((1-2 v)^{*} \varepsilon\right)-1=\mathrm{p}^{*}\left(\exp \left(\left(1-2 v_{\mathrm{p}}\right) * \varepsilon\right)-1\right)+(1-\mathrm{p}) *\left(\exp \left(\left(1-2 v_{\mathrm{m}}\right) * \varepsilon\right)-1\right) \quad$ Eq.7S

Assuming $\mathrm{p}=\mathrm{p}_{0} * \exp \left(-\beta^{*} \varepsilon\right)$, with $\beta$ denoting a shape factor describing the variation of the pore shape under strain, we note that $\beta>1-2 v_{p}$ for the self-consistency $\left(v=v_{m}\right)$ in the asymptotic limit $\varepsilon \rightarrow \infty$, where $\mathrm{p}_{0}$ is the pore volume fraction at vanishing strain $(\varepsilon=0)$.

Assuming for the relative volume change of the pores a model similar to that describing the Hoberman sphere, ${ }^{1 \mathrm{~S}-3 \mathrm{~S}}$ Eq. $6 \mathrm{~S}$ yields:

$\Delta \mathrm{V}_{\mathrm{p}} / \mathrm{V}_{\mathrm{p}}=\left(\mathrm{R}^{3}-\mathrm{r}^{3}\right) / \mathrm{r}^{3}=(\mathrm{R} / \mathrm{r})^{\wedge}\left(1-2 v_{\mathrm{p}}\right)-1$

Eq.8S

where $\mathrm{r}$ and $\mathrm{R}$ are the radius of the pore before and after expansion. Thus Eq. 8S predicts for a sphere which maintains its shape as it expands or collapses a Poisson's ratio $v_{\mathrm{p}}=-1$.

Finally, Eq. 7S for $\varepsilon \rightarrow 0$ and $v_{\mathrm{p}}=-1$, gives:

$v=\left(1-3 p-(1-p) *\left(1-2 * v_{m}\right)\right) / 2$ Eq.9S

that restitutes for $\mathrm{p}=0$ the Poisson's ratio of the matrix $\left(v_{\mathrm{m}}\right)$ while for $\mathrm{p}=1$ the Poisson ratio of the pores, here equal to -1 . For $\varepsilon \rightarrow \infty$, Eq.9S gives the Poisson's ratio of the matrix (i.e. $v_{\mathrm{m}}$ ). The same self-consistent asymptotic behaviors are guaranteed by Eq.7S for $v_{\mathrm{p}}=-1$ and $\beta>3$.

\section{Density}

The density of composite material $\left(\rho_{c}\right)$ can be defined as the ratio of weight of the composite material $\left(\mathrm{W}_{\mathrm{c}}\right)$ to the volume of the composite material $\left(\mathrm{V}_{\mathrm{c}}\right)$ and is expressed as $\rho_{\mathrm{c}}=\mathrm{W}_{\mathrm{c}} / \mathrm{V}_{\mathrm{c}}$ 


\section{WILEY-VCH}

that being $\mathrm{V}=\mathrm{W} / \rho$, can be written in terms of weight fraction as:

$1 / \rho_{\mathrm{c}}=1 / \rho_{\mathrm{f}} *\left(\mathrm{~W}_{\mathrm{f}} / \mathrm{W}_{\mathrm{c}}\right)+1 / \rho_{\mathrm{m}} *\left(\mathrm{~W}_{\mathrm{m}} / \mathrm{W}_{\mathrm{c}}\right)$

where $\mathrm{W}_{\mathrm{m}}\left(\rho_{\mathrm{m}}\right)$ and $\mathrm{W}_{\mathrm{f}}\left(\rho_{\mathrm{f}}\right)$ and are the weight fraction (density) of the matrix and the filler, respectively. Thus, in general, the density of the composite material in terms of weight fractions $\mathrm{w}_{\mathrm{i}}=\mathrm{W}_{\mathrm{i}} / \mathrm{W}_{\mathrm{c}}$ can be written as:

$\rho_{\mathrm{c}}=1 / \Sigma_{\mathrm{i}=1}^{\mathrm{n}}\left(\mathrm{w}_{\mathrm{i}} / \rho_{\mathrm{i}}\right)$.

The theoretical density values reported in Tab. 1S have been obtained from the weight fractions and density values reported in the Materials and Method section of the main text. The pore volume fraction $\left(\mathrm{p}_{0}\right)$ has been calculated from the sample $(\rho)$ and theoretical $\left(\rho_{\mathrm{c}}\right)$ densities from the mass balance $\rho=\left(1-\mathrm{p}_{0}\right)^{*} \rho_{\mathrm{c}}$.

Table 1S. Theoretical densities and pore volume fraction of the bionic composites.

\begin{tabular}{c|c|c}
\hline $\begin{array}{c}\text { Samples } \\
\text { (CNTs wt\%/GNPs wt\%) }\end{array}$ & $\begin{array}{c}\text { Theoretical density } \\
\left(\mathrm{g} / \mathrm{cm}^{3}\right)\end{array}$ & $\begin{array}{c}\text { Pore volume fraction } \\
\left(\mathrm{p}_{0}\right)\end{array}$ \\
\hline $0 / 0$ & 1.05 & 0.55 \\
\hline $1 / 0$ & 0.91 & 0.50 \\
\hline $0 / 1$ & 0.90 & 0.31 \\
\hline $0.5 / 0.5$ & 0.80 & 0.60 \\
\hline
\end{tabular}




\section{WILEY-VCH}

Table 2S. Density normalized mechanical characteristics of the prepared samples.

\begin{tabular}{c|c|c|c|c}
\hline $\begin{array}{c}\text { Samples } \\
(\mathrm{CNTs} w \mathrm{wt} / \mathrm{GNPs} \\
\text { wt\%) }\end{array}$ & $\begin{array}{c}\text { Young } \\
\text { modulus/Density } \\
(\mathrm{MPa}) /\left(\mathrm{g} / \mathrm{cm}^{3}\right)\end{array}$ & $\begin{array}{c}\text { Toughness/Density } \\
(\mathrm{MPa}) /\left(\mathrm{g} / \mathrm{cm}^{3}\right)\end{array}$ & $\begin{array}{c}\text { Fracture toughness/Density } \\
\left(\mathrm{MPa}^{*} \mathrm{~mm}^{1 / 2}\right) /\left(\mathrm{g} / \mathrm{cm}^{3}\right)\end{array}$ & $\begin{array}{c}\text { Bulk density } \\
\left(\mathrm{g} / \mathrm{cm}^{3}\right)\end{array}$ \\
\hline $0 / 0$ & $0.13 \pm 0.08$ & $0.09 \pm 0.07$ & $0.11 \pm 0.08$ & $1.04 \pm 0.06$ \\
\hline $1 / 0$ & $0.62 \pm 0.1$ & $0.05 \pm 0.05$ & $0.20 \pm 0.06$ & $0.91 \pm 0.06$ \\
\hline $0 / 1$ & $0.49 \pm 0.1$ & $0.08 \pm 0.03$ & $0.19 \pm 0.04$ & $0.90 \pm 0.04$ \\
\hline $0.5 / 0.5$ & $0.73 \pm 0.1$ & $0.09 \pm 0.02$ & $0.22 \pm 0.03$ & $0.79 \pm 0.04$ \\
\hline Bionic & & & & $0.48 \pm 0.06$ \\
\hline $0 / 0$ & $0.27 \pm 0.07$ & $0.21 \pm 0.07$ & $0.75 \pm 0.13$ & $0.46 \pm 0.04$ \\
\hline $1 / 0$ & $0.13 \pm 0.02$ & $0.15 \pm 0.02$ & $0.48 \pm 0.05$ & $0.62 \pm 0.03$ \\
\hline $0 / 1$ & $0.26 \pm 0.02$ & $0.26 \pm 0.02$ & $0.74 \pm 0.05$ & $0.32 \pm 0.03$ \\
\hline $0.5 / 0.5$ & $0.50 \pm 0.05$ & $0.22 \pm 0.03$ & $0.93 \pm 0.10$ & \\
\hline
\end{tabular}
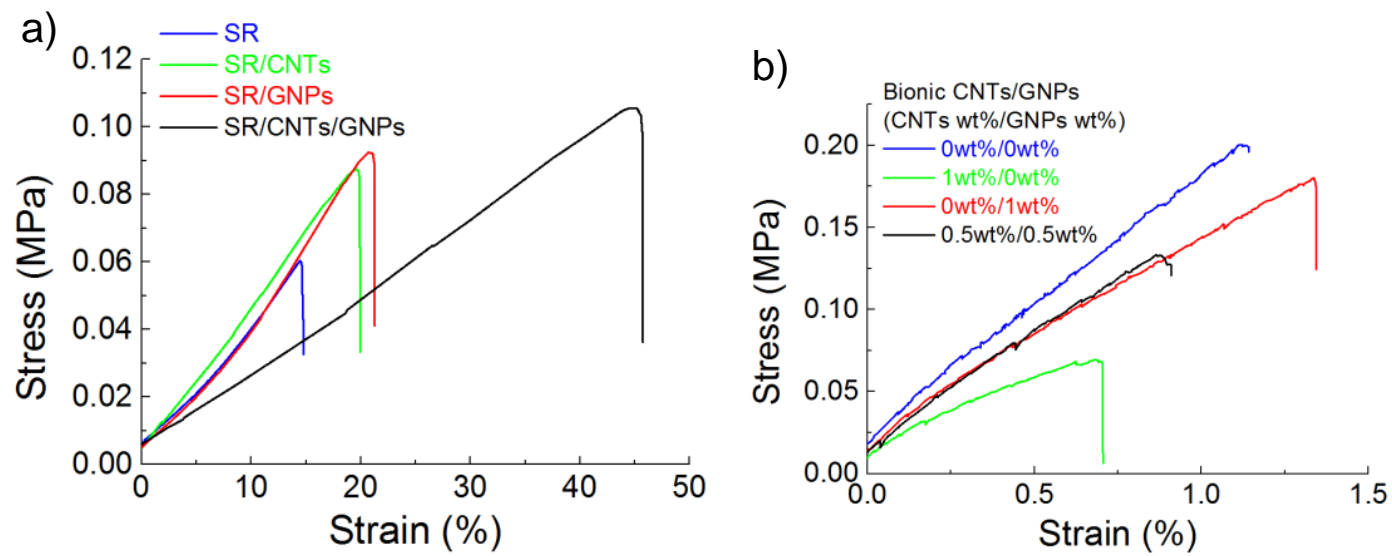

Figure 1S. Stress-strain curves of notched (a) conventional and (b) bionic composites. 


\section{WILEY-VCH}

Table 3S. Pore size and pore shape values obtained by the software analysis of the FESEM images of the bionic CNTs/GNPs samples (CNTs wt\%/GNPs wt\%). In the panel is reported the FESEM image $(515 \mu \mathrm{m} \times 417 \mu \mathrm{m})$ of the bionic SR/CNTs sample elaborated by the Image-J software for the calculation of the pore size and pore circularity.

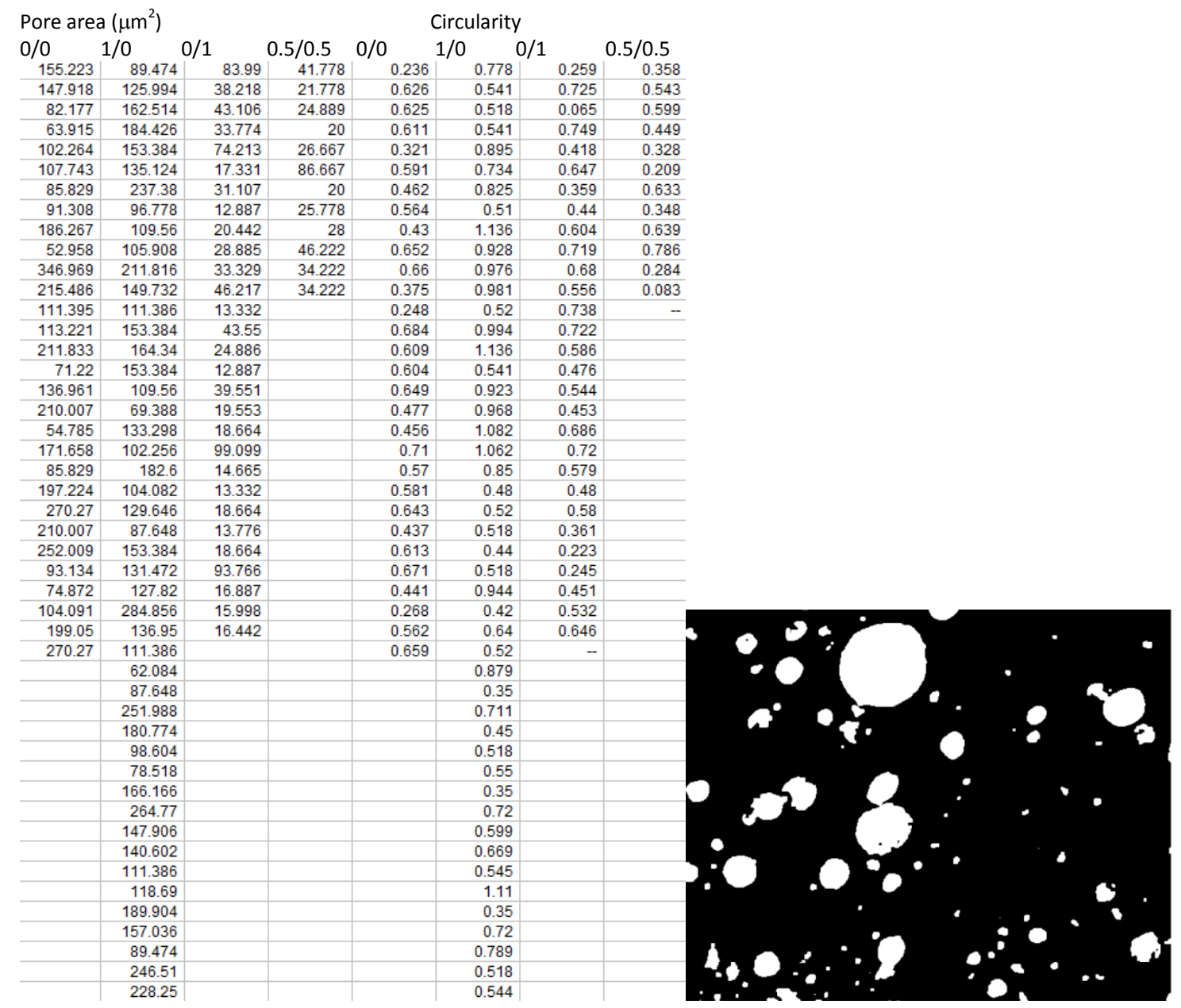




\section{WILEY-VCH}

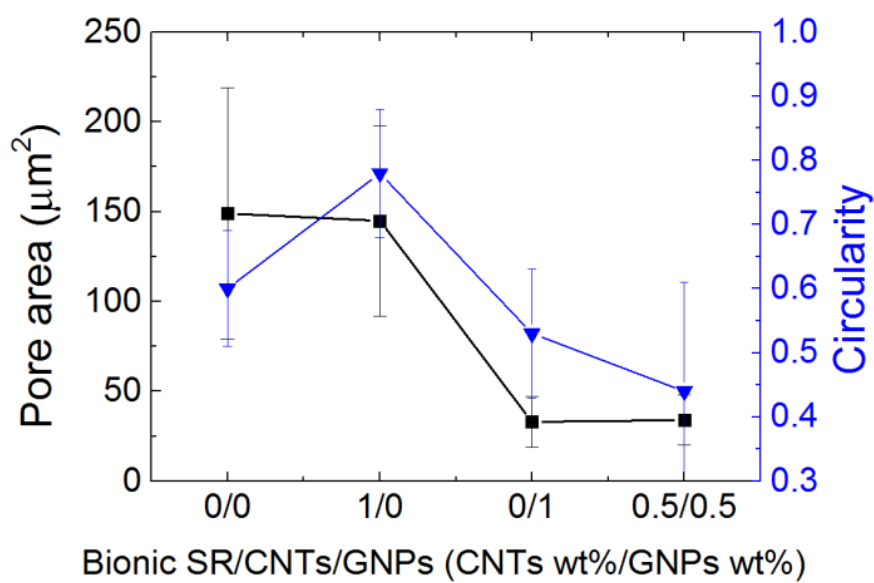

Figure 2S. Average pore size and average pore circularity of the bionic composites reported in Figures $1 \mathrm{c}-\mathrm{f}$.

Table 4S. Average values of pore size and pore shape of the bionic composites vs. their $\underline{\text { Poisson's ratios at } 0 \% \text { of strain and electrical resistance values. }}$

\begin{tabular}{c|c|c|c|c}
\hline $\begin{array}{c}\text { Bionic samples } \\
\text { (CNTs wt\%/GNPs wt\%) }\end{array}$ & $\begin{array}{c}\text { Pore size } \\
\left(\mu \mathrm{m}^{2}\right)\end{array}$ & $\underline{\text { Circularity }}$ & $\underline{\text { Poisson's ratio }}$ & $\begin{array}{c}\text { Electrical resistance } \\
\text { at strain 0\% }\end{array}$ \\
\hline$\underline{0 / 0}$ & $\underline{149 \pm 70}$ & $\underline{0.60 \pm 0.10}$ & $\underline{-0.49}$ & $=$ \\
\hline$\underline{1 / 0}$ & $\underline{145 \pm 53}$ & $\underline{0.70 \pm 0.10}$ & $\underline{0.14}$ & $\underline{7.39 \mathrm{E} 10}$ \\
\hline$\underline{0 / 1}$ & $\underline{33 \pm 14}$ & $\underline{0.53 \pm 0.10}$ & $\underline{-0.60}$ & $\underline{7.57 \mathrm{E} 10}$ \\
\hline$\underline{0.5 / 0.5}$ & $\underline{34 \pm 14}$ & $\underline{0.44 \pm 0.17}$ & $\underline{-0.75}$ & $\underline{7.20 \mathrm{E} 10}$ \\
\hline
\end{tabular}




\section{WILEY-VCH}

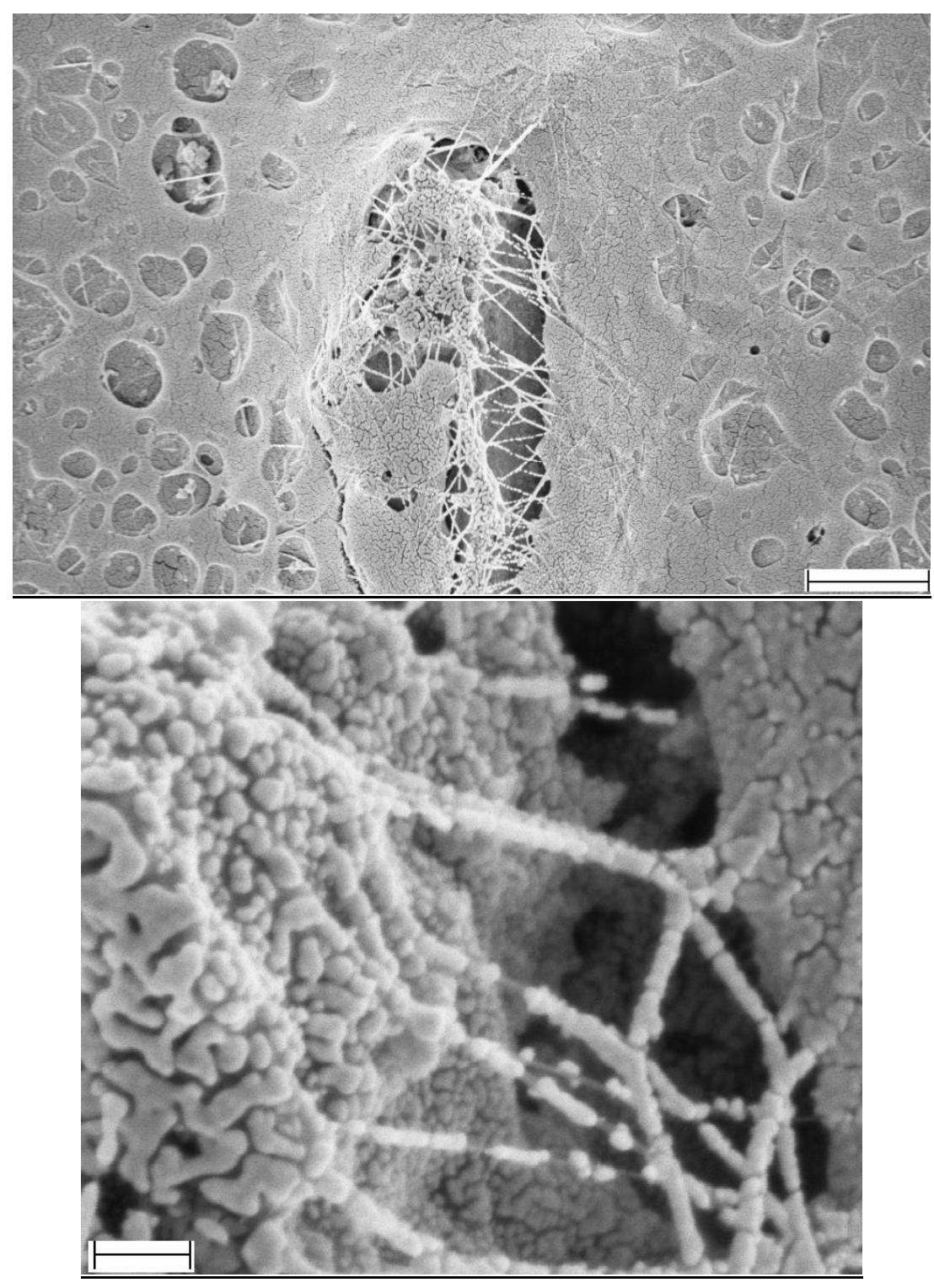

Figure 3S. FESEM images at different magnifications presenting the metallized fracture

surface of the bionic SR/CNTs composite showing the pull-out CNTs.

References

1S. J. Shim, Proc. Natl. Acad. Sci. U.S.A. 2012, 109, 5978.

3S. C. Hoberman, US Patent no. 4-942-700, (1991). 